\title{
Use of the modified Glasgow Coma Scale score to guide sequential invasive-noninvasive mechanical ventilation weaning in patients with AECOPD and respiratory failure
}

\author{
JIN-BO ZHANG ${ }^{1}$, JIN-QIANG ZHU ${ }^{1}$, LIE-XIANG CAO ${ }^{1}$, XIAO-HONG JIN ${ }^{1}$, LI-LI CHEN ${ }^{1}$, YU-KANG SONG ${ }^{1}$, \\ SHI-FANG ZHOU ${ }^{2}$, JI-HONG MA ${ }^{3}$, HUI FU ${ }^{1}$, JIN-ZHONG XU ${ }^{1}$, MEI-PING DONG ${ }^{1}$, LAI-CHAO YAN ${ }^{1}$, \\ XIAN-DAN WU ${ }^{1}$, HUI-PING WANG ${ }^{1}$, JUN-YANG ZHOU ${ }^{1}$ and YAN-QIU WANG ${ }^{1}$
}

\author{
${ }^{1}$ Emergency Intensive Care Unit, Wenling Hospital Affiliated to Wenzhou Medical University, The First People's Hospital of \\ Wenling, Wenling, Zhejiang 317500; ${ }^{2}$ Department of Emergency Care, Changsha Central Hospital, Changsha, Hunan 410004; \\ ${ }^{3}$ Intensive Care Unit, First Affiliated Hospital of Wenzhou Medical University, Wenling, Zhejiang 325000, P.R. China
}

Received December 16, 2019; Accepted May 7, 2020

DOI: $10.3892 /$ etm.2020.8884

\begin{abstract}
Sequential invasive-noninvasive ventilation (NIV) improves the outcomes of patients with respiratory failure caused by acute exacerbation of chronic obstructive pulmonary disease (AECOPD); however, there is no clear consensus on the optimal timing of the switch to sequential invasive-NIV in these patients. In the present study, a potential role for the modified Glasgow Coma Scale (GCS) score to guide sequential weaning was investigated. Patients with AECOPD and respiratory failure were prospectively recruited from three study centers (Wenling Hospital Affiliated to Wenzhou Medical University, the First Affiliated Hospital of Wenzhou Medical University and Changsha Central Hospital) between January 1st 2016 and December 31st 2018. Patients were randomly assigned to group A and B, with the switching point for sequential weaning strategy in the two groups being a modified GCS score $\geq 13$
\end{abstract}

Correspondence to: Dr Jin-Qiang Zhu, Emergency Intensive Care Unit, Wenling Hospital Affiliated to Wenzhou Medical University, The First People's Hospital of Wenling, 333 Chuan An South Road, Wenling, Zhejiang 317500, P.R. China

E-mail: zhangjinbo0661@163.com

Abbreviations: AECOPD, acute exacerbation of chronic obstructive pulmonary disease; APACHE II, Acute Physiology and Chronic Health Enquiry; ABG, arterial blood gas; BMI, body mass index; COPD, chronic obstructive pulmonary disease; GCS, Glasgow Coma Scale; HR, heart rate; ICU, intensive care unit; IMV, invasive mechanical ventilation; MBP, mean blood pressure; NIV, noninvas; $\mathrm{OI}$, oxygenation index; $\mathrm{PaCO}_{2}$, partial pressure of arterial $\mathrm{CO}_{2} ; \mathrm{PaO}$, partial pressure of arterial oxygen; PIC, pulmonary infection control; RR, respiratory rate; SBT, spontaneous breathing trial; VAP, ventilator-associated pneumonia

Key words: acute exacerbation of chronic obstructive pulmonary disease, respiratory failure, invasive mechanical ventilation, noninvasive ventilation, Glasgow Coma Scale and 10 points, respectively. Each group included 240 patients. Baseline demographic characteristics were comparable in the two groups. The duration of invasive mechanical ventilation (IMV) in group A was significantly shorter than that in group B. However, there were no significant between-group differences with respect to the incidence of re-intubation, ventilator-associated pneumonia, in-hospital mortality or the length of hospital stay. Use of a modified GCS score $\geq 13$ as the switching point for sequential invasive-NIV may help decrease the duration of IMV in patients with AECOPD and respiratory failure.

\section{Introduction}

Chronic obstructive pulmonary disease (COPD) accounts for a major proportion of global morbidity and mortality (1). An estimated 384 million people aged $\geq 30$ years are believed to be affected by COPD [global prevalence: $11.7 \%$ (8.4-15.0\%)] (1). Hospitalization in the intensive care unit (ICU) is required for $\sim 12-18 \%$ of patients with COPD due to acute exacerbations (2), with the mortality rate of these patients approaching $15 \%$ (3). Invasive mechanical ventilation (IMV) is the primary choice of treatment for 5.9-26.0\% of patients with acute exacerbation of COPD (AECOPD), especially comatosed patients $(4,5)$. Despite the development of protective lung ventilation strategies and the optimization of treatment for respiratory failure, the reported incidence of AECOPD-induced respiratory failure is $>24.5 \%$ (6). Prolonged IMV may lead to ventilator-associated pneumonia (VAP), ventilator-associated lung injury and difficulty in weaning from mechanical ventilation, which can exacerbate respiratory distress and necessitate the continuation of invasive ventilation $(7,8)$. Prolonged endotracheal intubation can also lead to airway injury, tracheoesophageal fistula and other potentially serious complications (7). Furthermore, long-term endotracheal intubation reduces the quality of life of patients and leads to a poor prognosis; therefore, timely weaning from mechanical ventilation is an important factor for the use of IMV (8).

Sequential invasive-noninvasive ventilation (NIV) is a widely used strategy to decrease the duration of IMV. 
However, there is no clear consensus on the optimal switching point (5). Excellent results have been reported with the use of a pulmonary infection control (PIC) window as the switching point for the implementation of a sequential ventilation strategy (9). However, the PIC approach is mostly based on $\mathrm{x}$-ray imaging findings and does not take into account the lag time between the appearance of x-ray findings and clinical manifestations (9).

Indeed, patients with AECOPD and respiratory failure who exhibit a high level of consciousness and cooperation, typically benefit from NIV, while impaired consciousness has been identified as a risk factor for extubation failure $(10,11)$. Previous studies have investigated the use of a modified Glasgow Coma Scale (GCS) score for evaluating the level of consciousness of intubated patients $(12,13)$. the modified GCS score was found to be a more objective, quantitative measure of the overall clinical condition. The present study aimed to compare two weaning strategies entailing the use of different levels of modified GCS score (13 vs. 10 points) as the switching point for the sequential invasive-NIV in patients with AECOPD.

\section{Materials and methods}

Study participants. In this prospective, randomized, controlled study, consecutive patients with AECOPD who received intubation for respiratory failure at the ICU of 3 hospitals (Wenling Hospital Affiliated to Wenzhou Medical University, the First Affiliated Hospital of Wenzhou Medical University and Changsha Central Hospital) were recruited between January 1st 2016 and December 31st 2018. The Ethics Committee of the Wenling Hospital Affiliated to the Wenzhou Medical University approved the research protocol for this study. Written informed consent was obtained from all patients prior to their enrolment.

The inclusion criteria were as follows: i) Age $\geq 18$ years; ii) patients who received IMV for respiratory failure; iii) patients who met the COPD diagnostic criteria in the 2017 guidelines of the Global Initiative for Chronic Obstructive Lung Disease (14); iv) partial pressure of arterial oxygen $\left(\mathrm{PaO}_{2}\right)$ and $\mathrm{CO}_{2}\left(\mathrm{PaCO}_{2}\right)$ in arterial blood gas (ABG) analysis <60 mmHg; and v) no absolute contraindications to NIV (15). The exclusion criteria were as follows: i) Acute stroke, acute pulmonary embolism, cardiogenic pulmonary edema or other causes of acute respiratory failure; ii) death within 3 days of admission; iii) active upper gastrointestinal bleeding; iv) treatment discontinuation; or v) readmission to the ICU $<3$ months following enrolment with the study. A total of 283 patients qualified for the inclusion criteria, with 240 patients finally included after screening. A total of 141 men and 99 women were included in the current study (mean age, 55.3 \pm 9.1 years; age range, 31-86 years). All the enrolled patients were randomly assigned to groups $\mathrm{A}$ and $\mathrm{B}$, and there were no significant between-group differences with respect to sex, age or body mass index (BMI). A schematic illustration of the study design and patient-selection criteria is presented in Fig. 1.

Groups and definitions of the modified GCS score. Using a random number table, subjects were randomly assigned to group $\mathrm{A}$ and $\mathrm{B}$, based on the use of modified GCS score $\geq 13$ or 10 points, respectively, as the switching point for sequential weaning strategy. The detailed definition of the modified GCS score is shown in Table I.

Treatment protocols. All subjects received the following treatments: Anti-infective agents, antispasmodics, glucocorticoids (methylprednisolone, 40-80 mg/day), anti-inflammatory agents, expectorants, nutritional support and sedatives, as well as measures for maintaining internal homeostasis. Dexmedetomidine was used as the first-line sedative, which was administered as an intravenous bolus infusion over $10 \mathrm{~min}$ at a dose of $1 \mu \mathrm{g} / \mathrm{kg}$, followed by $0.2-0.8 \mu \mathrm{g} / \mathrm{kg} / \mathrm{h}$ using a micro-pump. The infusion rate was titrated to maintain a Richmond Agitation-Sedation score between-2 and +1 (16). The daily neurological wake-up test consisted of 3 components: Eye-opening in response to verbal command, eye tracking and shaking hands-on instruction if the sedation was in the target range. If the above criteria were not met, the sedative dose was adjusted until the target was reached.

Weaning protocol. The parameters of mechanical ventilation were calibrated based on the results of the ABG analysis and the disease course. In Group A, patients were ventilated with synchronized intermittent mandatory ventilation mode, with the addition of pressure support ventilation or the assist/control mode. The respiratory rate (RR) was set at $13-18$ cycles/min and tidal volume at $8-10 \mathrm{ml} / \mathrm{kg}$ to maintain the arterial partial pressure of $\mathrm{CO}_{2}\left(\mathrm{PaCO}_{2}\right)$ at $35-50 \mathrm{mmHg}$. The inspired oxygen fraction and positive end-expiratory pressure were adjusted to maintain arterial oxygen saturation of $\geq 90 \%$. IMV was switched to NIV (Philips Medical Systems, Inc.) if the patients remained stable for $3 \mathrm{~h}$ after achieving the target modified GCS score in their respective groups (13 points in Group A and 10 points in Group B). Subsequently, the spontaneous/timed mode was applied, with an inspiratory positive airway pressure of $12-14 \mathrm{~cm} \mathrm{H}_{2} \mathrm{O}$ and expiratory positive airway pressure of $5 \mathrm{cmH}_{2} \mathrm{O}$, which were gradually increased to the appropriate level within 5-20 min of switching to NIV.

Data collection and outcomes. Data pertaining to the baseline characteristics and indices at admission were collected, including the Acute Physiology and Chronic Health Enquiry score, modified GCS score, mean arterial blood pressure (MBP) and oxygenation index (OI). Indices such as the MBP, $\mathrm{OI}$, heart rate (HR), RR and results of the $\mathrm{ABG}$ analysis were also measured before and $3 \mathrm{~h}$ after weaning to NIV. The primary outcome was the duration of invasive ventilation. Other outcomes included the incidence of VAP, re-intubation rate, in-hospital mortality and the length of hospital stay.

Statistical analysis. All statistical analyses were conducted using SPSS 25.0 (for Windows; IBM Corp.). Continuous variables are expressed as the mean $\pm(\mathrm{SD})$. Between-group differences with respect to continuous variables were assessed using the unpaired Student's t-test. Categorical variables are expressed as ratios and between-group differences were assessed using the $\chi^{2}$ test. All statistical analyses were two-sided, and $\mathrm{P}<0.05$ was considered to indicate a statistically significant difference. 
Table I. Modified GCS.

\begin{tabular}{llll}
\hline Score & \multicolumn{1}{c}{ Eye } & \multicolumn{1}{c}{ Verbal } & \multicolumn{1}{c}{ Motor } \\
\hline 1 & Does not open eyes & No response to speech & Makes no movements \\
2 & Opens eyes in response to painful stimuli & Response to loudly call & Extension to painful stimuli \\
3 & Opens eyes in response to voice & Understanding error & Abnormal flexion to painful stimuli \\
4 & Opens eyes spontaneously & Slow understanding of speech & Flexion/withdrawal from painful stimuli \\
5 & N/A & Correct understanding of speech & Localizes painful stimuli \\
6 & N/A & N/A & Obeys commands \\
\hline
\end{tabular}

The modified GCS evaluates 3 parameters: Eye, verbal and motor responses. The separate scores of these 3 parameters as well as their combined scores are considered. The lowest possible GCS, the sum, score is 3 (deep coma or death), whereas the highest is 15 (fully conscious person). N/A, not applicable. GCS, Glasgow Coma Scale.

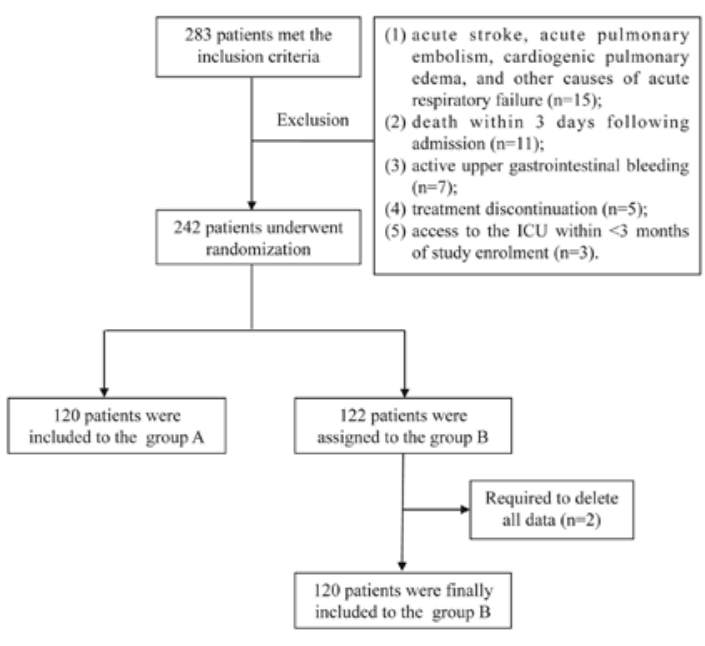

Figure 1. Schematic illustration of the study design and patient-selection criteria. ICU, intensive care unit.

\section{Results}

Baseline characteristics. A total of 240 patients [141 men and 99 women; mean age \pm SD: 55.3 \pm 9.1 (range 31-86) years] qualified the study selection criteria and were enrolled; of these 120 patients each were randomly assigned to groups A and B. The baseline demographic characteristics of patients in the two groups were comparable at the time of randomization (Table II). There were no significant between-group differences with respect to sex, age or BMI. There were no significant differences with respect to concomitant diseases such as cardiovascular diseases, cerebrovascular diseases, diabetes and chronic kidney disease. Likewise, there were no significant between-group differences with respect to MBP, HR, respiratory rate, OI or arterial blood analysis at admission.

Safety and efficiency of sequential weaning as guided by two different modified GCS scores. Patients in groups A and $B$ underwent extubation followed by sequential NIV upon reaching modified GCS score of $\geq 13$ points and 10 , respectively. There were no significant between-group differences with respect to OI, MBP, $\mathrm{PaO}_{2}$ and $\mathrm{PaCO}_{2}(\mathrm{P}>0.05$; Table III) both before extubation and at $3 \mathrm{~h}$ after extubation.

Primary and secondary outcomes. The duration of IMV in group A was significantly shorter than that in Group B (Table IV). However, there were no significant between-group differences with respect to the incidence rate of re-intubation, VAP, in-hospital mortality or the length of hospital stay.

\section{Discussion}

The present study was a randomized clinical trial conducted at three ICUs in Eastern China. It was found that sequential NIV, guided by a modified GCS score, was an effective and safe weaning strategy in AECOPD patients undergoing IMV. Moreover, the use of a modified GCS score $\geq 13$ points as the criterion to switch to sequential NIV, decreased the duration of invasive ventilation in ICU patients with AECOPD-induced respiratory failure, as compared to a modified GCS score $\geq 10$ points. However, there were no significant differences with respect to the incidence of re-intubation, VAP, hospital mortality or the length of hospital stay.

The identification of a timely and optimal 'switching point' is the key to an effective sequential ventilation strategy (9). Use of spontaneous breathing trial (SBT) to predict the successful withdrawal of the IMV is recommended by the European Respiratory Society, the American Thoracic Society, the European Society of Intensive Care Medicine, the Society of Critical Care Medicine and the Société de Réanimation de langue Française (17). It is believed that the duration of SBT should be between 30-120 min (18); however, it is not clear whether the same duration is applicable to patients who require mechanical ventilation because of different diseases. It has been found that SBT may cause a delay in weaning and may also cause VAP, which in turn may contribute to increased mortality (19). In China, the switching point is mainly the PIC window, as proposed by the Beijing Institute of Respiratory Diseases (20), while other countries, such as England, have employed a 48-h window for this purpose (21). However, both of these strategies have certain limitations. The former ignores the time-lag between the appearance of imaging findings and the onset of clinical manifestations, and does not take 
Table II. Baseline characteristics of the study population.

\begin{tabular}{|c|c|c|c|c|}
\hline Variables & Group A, n=120 & Group $B, n=120$ & $\mathrm{t} / \chi^{2}$ value & P-value \\
\hline Male & $75(62.5 \%)$ & $66(55.0 \%)$ & $-0.464^{\mathrm{a}}$ & 0.496 \\
\hline Age, years & $57 \pm 8$ & $54 \pm 10$ & $0.693^{\mathrm{b}}$ & 0.489 \\
\hline BMI, $\mathrm{kg} / \mathrm{m}^{2}$ & $21.87 \pm 3.79$ & $20.33 \pm 2.51$ & $0.702^{\mathrm{b}}$ & 0.484 \\
\hline \multicolumn{5}{|l|}{ Primary diseases } \\
\hline Cardiovascular disease & $19(15.83 \%)$ & $22(18.33 \%)$ & $0.265^{\mathrm{a}}$ & 0.607 \\
\hline Cerebrovascular disease & $25(20.83 \%)$ & $17(14.17 \%)$ & $1.847^{\mathrm{a}}$ & 0.174 \\
\hline Diabetes & $16(13.33 \%)$ & $22(18.33 \%)$ & $1.126^{\mathrm{a}}$ & 0.289 \\
\hline Chronic kidney disease & $9(7.50 \%)$ & $6(5.00 \%)$ & $0.640^{\mathrm{a}}$ & 0.424 \\
\hline APACHE II & $23.89 \pm 4.32$ & $22.90 \pm 3.79$ & $0.768^{\mathrm{b}}$ & 0.452 \\
\hline $\mathrm{MBP}, \mathrm{mmHg}$ & $102.59 \pm 20.03$ & $98.25 \pm 21.92$ & $1.251^{\mathrm{b}}$ & 0.224 \\
\hline $\mathrm{OI}, \mathrm{mmHg}$ & $158.26 \pm 32.82$ & $161.13 \pm 26.57$ & $0.42^{\mathrm{b}}$ & 0.813 \\
\hline Heart rate, bpm & $87.23 \pm 15.81$ & $85.62 \pm 17.19$ & $0.853^{\mathrm{b}}$ & 0.392 \\
\hline Respiratory rate, bpm & $21.21 \pm 5.14$ & $19.68 \pm 4.17$ & $1.35^{\mathrm{b}}$ & 0.187 \\
\hline $\mathrm{pH}$ value & $7.21 \pm 1.51$ & $7.25 \pm 1.90$ & $0.148^{\mathrm{b}}$ & 0.881 \\
\hline $\mathrm{PaO} 2, \mathrm{mmHg}$ & $58.35 \pm 11.24$ & $56.09 \pm 9.83$ & $1.649^{\mathrm{b}}$ & 0.105 \\
\hline $\mathrm{PaCO} 2, \mathrm{mmHg}$ & $85.19 \pm 17.53$ & $82.39 \pm 18.07$ & $1.276^{\mathrm{b}}$ & 0.207 \\
\hline
\end{tabular}

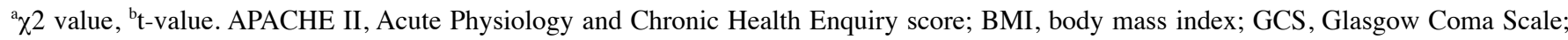
$\mathrm{MBP}$, mean blood pressure; OI, oxygenation index; $\mathrm{PaCO}_{2}$, arterial partial pressure of $\mathrm{CO}_{2} ; \mathrm{PaO}_{2}$, arterial partial pressure of $\mathrm{O}_{2}$.

Table III. Indices before and $3 \mathrm{~h}$ after extubation.

Comparison between

Group A and B

\begin{tabular}{|c|c|c|c|c|c|}
\hline \multirow[b]{2}{*}{ Time } & \multirow[b]{2}{*}{ Variables } & \multirow[b]{2}{*}{ Group A } & \multirow[b]{2}{*}{ Group B } & \\
\hline & & & & $\mathrm{t}$ value & P-value \\
\hline \multirow[t]{4}{*}{ Before extubation } & $\mathrm{MBP}, \mathrm{mmHg}$ & $101.50 \pm 19.53$ & $105.37 \pm 17.63$ & 0.672 & 0.506 \\
\hline & $\mathrm{OI}, \mathrm{mmHg}$ & $222.16 \pm 40.83$ & $225.25 \pm 43.09$ & 1.298 & 0.192 \\
\hline & $\mathrm{PaO}_{2}, \mathrm{mmHg}$ & $88.65 \pm 19.26$ & $87.58 \pm 17.30$ & 1.034 & 0.316 \\
\hline & $\mathrm{PaCO}_{2}, \mathrm{mmHg}$ & $44.26 \pm 12.64$ & $43.60 \pm 10.25$ & 0.685 & 0.493 \\
\hline \multirow{4}{*}{$\begin{array}{l}3 \text { hours after extubation and } \\
\text { noninvasive ventialtion }\end{array}$} & $\mathrm{MBP}, \mathrm{mmHg}$ & $102.86 \pm 22.69$ & $102.56 \pm 20.35$ & 0.857 & 0.389 \\
\hline & $\mathrm{OI}, \mathrm{mmHg}$ & $210.29 \pm 37.47$ & $213.06 \pm 37.31$ & 0.831 & 0.408 \\
\hline & $\mathrm{PaO}_{2}, \mathrm{mmHg}$ & $81.55 \pm 21.63$ & $84.63 \pm 22.65$ & 0.658 & 0.514 \\
\hline & $\mathrm{PaCO}_{2}, \mathrm{mmHg}$ & $45.75 \pm 16.14$ & $48.32 \pm 11.74$ & 0.693 & 0.491 \\
\hline
\end{tabular}

MBP, mean blood pressure; OI, oxygenation index; $\mathrm{PaO}_{2}$, partial pressure of arterial oxygen; $\mathrm{PaCO}_{2}$, partial pressure of arterial $\mathrm{CO}_{2}$.

Table IV. Primary and secondary outcomes.

\begin{tabular}{|c|c|c|c|c|}
\hline Variables & Group A $(n=120)$ & Group B $(n=120)$ & $\mathrm{t} / \chi^{2}$ value & $\mathrm{P}$-value \\
\hline Hospital mortality (n, \%) & $8(6.67 \%)$ & $10(8.33 \%)$ & $0.240^{\mathrm{a}}$ & 0.624 \\
\hline Duration of IMV (days) & $4.13 \pm 1.04$ & $4.96 \pm 0.69$ & $2.003^{\mathrm{b}}$ & 0.045 \\
\hline Incidence of VAP $(\mathrm{n}, \%)$ & $3(7.5)$ & $6(15.0)$ & $1.127^{\mathrm{a}}$ & 0.288 \\
\hline Re-intubation (n, \%) & $2(5.0)$ & $5(12.5)$ & $1.409^{\mathrm{a}}$ & 0.235 \\
\hline Length of hospital stay (days) & $15.85 \pm 3.87$ & $17.16 \pm 5.02$ & $1.485^{\mathrm{b}}$ & 0.152 \\
\hline
\end{tabular}

${ }^{a} \chi^{2}$ value, ${ }^{b} t$-value. IMV, invasive mechanical ventilation; VAP, ventilator-associated pneumonia.

account of noninfective factors. The latter strategy overlooks inter-individual variability among patients.
There are 3 prerequisites for the application of sequential NIV: A high level of consciousness, a certain degree of 
cooperation and appropriate compliance; therefore, the changes in consciousness should be factored while considering the switch to noninvasive respiratory support (22). The main causes of AECOPD-induced respiratory failure are pulmonary infection, ventilatory insufficiency or respiratory muscle fatigue (9). Therefore, the level of consciousness tends to vary with the development, exacerbation or improvement of AECOPD (22). The GCS score is widely used for the assessment of awareness. It is an objective measure for the dynamic assessment of the overall and physical condition of patients with COPD with severe respiratory failure at each stage of the disease (23). Patients with endotracheal intubation cannot speak even if they are conscious (24). Therefore, the GCS scoring system has been improved by modifying the correct response instead of using speech (23). A previous study found that it was safe and feasible to initiate sequential NIV if modified GCS score $\geq 13$ points, and that it was acceptable to stablely maintain for $3 \mathrm{~h} \mathrm{(25).} \mathrm{The}$ sequential ventilation strategy was found to reduce the discomfort, restlessness and pain induced by intubation, and increased patient trust and coordination. This resulted in a higher rate of successful weaning, lower incidence of re-intubation and shorter length of hospital stay. Chen et al (26) found that the use of modified GCS score $\geq 10$ points was a more suitable criterion for initiating the switch to sequential invasive-non-invasive weaning. In the present study, the two weaning strategies, which were guided respectively by 'improved GCS $\geq 13$ points' and 'improved GCS $\geq 10$ points', were compared. The use of the modified GCS score $\geq 13$ points as the criterion to switch to sequential NIV was associated with a shorter duration of invasive ventilation in ICU patients with AECOPD-induced respiratory failure as compared to using the modified GCS score $\geq 10$ points.

In conclusion, using a improvement of the modified GCS score $\geq 13$ points as the switching point for sequential invasive-NIV may significantly improve the prognosis of patients with AECOPD with respiratory failure.

\section{Acknowledgements}

Not applicable.

\section{Funding}

This study was supported by the Wenling Science and Technology Bureau Project (grant no. 2014C31BA0032).

\section{Availability of data and materials}

The datasets used and/or analyzed during the present study are available from the corresponding author on reasonable request.

\section{Authors' contributions}

JBZ and JQZ designed/performed most of the investigation, data analysis and wrote the manuscript. LXC, XJ and LLC provided statistics assistance. YS, SZ, JM, HF, JX, MD, LY, $\mathrm{XW}, \mathrm{HW}, \mathrm{JYZ}$ and YW contributed to interpretation of the data and analyses. All of the authors have read and approved the manuscript.

\section{Ethics approval and consent to participate}

The Ethics Committee of the Wenling Hospital Affiliated to the Wenzhou Medical University approved the research protocol for this study. Written informed consent was obtained from all patients prior to their enrolment.

\section{Patient consent for publication}

Not applicable.

\section{Competing interests}

The authors declare that they have no competing interests.

\section{References}

1. Adeloye D, Chua S, Lee C, Basquill C, Papana A, Theodoratou E, Nair H, Gasevic D, Sridhar D, Campbell H, et al: Global and regional estimates of COPD prevalence: Systematic review and meta-analysis. J Glob Health 5: 020415, 2015.

2. Rothberg MB, Pekow PS, Maureen L, Oren B, Skiest DJ and Lindenauer PK: Antibiotic therapy and treatment failure in patients hospitalized for acute exacerbations of chronic obstructive pulmonary disease. JAMA 303: 2035-2042, 2010.

3. Afessa B, Morales I, Scanlon PD and Peters SG: Prognostic factors, clinical course, and hospital outcome of patients with chronic obstructive pulmonary disease admitted to an intensive care unit for acute respiratory failure. Crit Care Med 30: 1610-1615, 2002.

4. Plant PK, Owen JL and Elliott MW: Early use of non-invasive ventilation for acute exacerbations of chronic obstructive pulmonary disease on general respiratory wards: A multicentre randomised controlled trial. Lancet 355: 1931-1935, 2000.

5. Stefan MS, Shieh MS, Pekow PS, Hill N, Rothberg MB and Lindenauer PK: Trends in mechanical ventilation among patients hospitalized with acute exacerbations of COPD in the United States, 2001 to 2011. Chest 147: 959-968, 2015.

6. Ai-Ping C,Lee KH and Lim TK: In-hospital and 5-year mortality of patients treated in the ICU for acute exacerbation of COPD: A retrospective study: Chest 128: 518-524, 2005.

7. Michelle B, Kashiouris MG and Ognjen G: Ventilator-induced lung injury: Minimizing its impact in patients with or at risk for ARDS. Respir Care 58: 927-934, 2013.

8. Forel JM, Voillet F, Pulina D, Gacouin A, Perrin G, Barrau K, Jaber S, Arnal JM, Fathallah M and Auquier P: Ventilator-associated pneumonia and ICU mortality in severe ARDS patients ventilated according to a lung-protective strategy. Crit Care 16: R65, 2012.

9. Lv Y, Lv Q, Lv Q and Lai T: Pulmonary infection control window as a switching point for sequential ventilation in the treatment of COPD patients: A meta-analysis. Int J Chron Obstruct Pulmon Dis 12: 1255-1267, 2017.

10. Frutos-Vivar F, Esteban A, Apezteguia C, González M, Arabi Y, Restrepo MI, Gordo F, Santos C, Alhashemi JA, Pérez FJ, et al: Outcome of reintubated patients after scheduled extubation. J Crit Care 26: 502-509, 2011.

11. Huang CT and Yu CJ: Conventional weaning parameters do not predict extubation outcome in intubated subjects requiring prolonged mechanical ventilation. Respir Care 58: 1307-1314, 2013.

12. Hendra KP, Bonis PA and Joyce-Brady M: Development and prospective validation of a model for predicting weaning in chronic ventilator dependent patients. BMC Pulm Med 3: 3, 2003.

13. Wu YK, Kao KC, Hsu KH, Hsieh MJ and Tsai YH: Predictors of successful weaning from prolonged mechanical ventilation in Taiwan. Respir Med 103: 1189-1195, 2009.

14. Vogelmeier CF, Criner GJ, Martinez FJ, Anzueto A, Barnes PJ, Bourbeau J, Celli BR, Chen R, Decramer M, Fabbri LM, et al: Global strategy for the diagnosis, management, and prevention of chronic obstructive lung disease 2017 report. GOLD Executive Summary. Am J Respir Crit Care Med 195: 557-582, 2017. 
15. Ornico SR, Lobo SM, Sanches HS, Deberaldini M, Tófoli LT, Vidal AM, Schettino GP, Amato MB, Carvalho CR and Barbas CS: Noninvasive ventilation immediately after extubation improves weaning outcome after acute respiratory failure: A randomized controlled trial. Crit Care 17: R39-R39, 2013.

16. Vasilevskis EE, Pandharipande PP, Graves AJ, Shintani A, Tsuruta R, Ely EW and Girard TD: Validity of a modified sequential organ failure assessment score using the richmond agitation-sedation scale. Crit Care Med 44: 138-146, 2016.

17. Boles JM, Bion J, Connors A, Herridge M, Marsh B, Melot C, Pearl R, Silverman H, Stanchina M, Vieillard-Baron A and Welte T: Weaning from mechanical ventilation. Eur Respir J 29: 1033-1056, 2007.

18. Burns KEA, Karim HMR and Esquinas AM: Characteristic of Subjects Who Fail a 120 -minute spontaneous breathing trial: When minutes are taken into account. Respir Care 64: 114, 2019.

19. Kallet RH: Ventilator bundles in transition: From prevention of ventilator-associated pneumonia to prevention of ventilator-associated events. Respir Care 64: 994-1006, 2019.

20. Luo Z, Zhan Q and Wang C: Noninvasive positive pressure ventilation is required following extubation at the pulmonary infection control window: A prospective observational study. Clin Respir J 8: 338-349, 2014

21. Perkins GD, Mistry D, Gates S, Gao F, Snelson C, Hart N, Camporota L, Varley J, Carle C, Paramasivam E, et al: Effect of protocolized weaning with early extubation to noninvasive ventilation vs invasive weaning on time to liberation from mechanical ventilation among patients with respiratory failure: The breathe randomized clinical trial. JAMA 320: 1881-1888, 2018.
22. Briones Claudett KH, Briones Claudett M, Chung Sang Wong M, Nuques Martinez A, Soto Espinoza R, Montalvo M, Esquinas Rodriguez A, Gonzalez Diaz G and Grunauer Andrade M: Noninvasive mechanical ventilation with average volume assured pressure support (AVAPS) in patients with chronic obstructive pulmonary disease and hypercapnic encephalopathy. BMC Pulm Med 13: 12, 2013.

23. Ciftci F, Ciledag A, Erol S, Oz M, Acar D and Kaya A: Evaluation of the feasibility of average volume-assured pressure support ventilation in the treatment of acute hypercapnic respiratory failure associated with chronic obstructive pulmonary disease: A pilot study. J Crit Care 39: 232-237, 2017.

24. Moskopp D, Stahle C and Wassmann H: Problems of the Glasgow Coma Scale with early intubated patients. Neurosurg Rev 18: 253-257, 1995.

25. Zhang J, Zhu J, Zhou S, Ma J, Fu H, Song Y, Xu J, Cao L, Dong $\mathrm{M}$, Yan $\mathrm{L}$, et al: Noninvasive mechanical ventilation in the weaning of AECOPD patients with respiratory failure: Modified glasgow coma scale score $\geq 13$ as the switching point. Int J Clinical Exp Med 12: 7808-7813, 2019.

26. Chen XLaW: Clincial investigation of modified GCS $\geq 10$ points as the switching point of sequantial sequential invasive-noninvasive mechanical ventilation weaning in AECOPD patients with respiratory failure. Chong Qing Yi Xue 45: 1381-1383, 2016 (In Chinese).

This work is licensed under a Creative Commons

Attribution-NonCommercial-NoDerivatives 4.0 International (CC BY-NC-ND 4.0) License. 